Ann. Biol. anim. Bioch. Biophys., I971, 11 (4), 625-643.

\title{
REPARTITION DES ÉLECTROLYTES ET DU GLUCOSE DANS L'EEUF CONSERVÉ SOUS 0 OU 2 P. 100 DE GAZ CARBONIQUE
}

\author{
B. SAUVEUR \\ avec la collaboration technique de J. RocARD \\ Station de Recherches avicoles, \\ Centre"de Recherches de Tours, I. N. R. A., \\ 37 - Nouzilly
}

RÉSUMÉ

Des œufs ont été conservés pendant 2, I 2 et 20 jours sous 2 p. Ioo (lot $\mathrm{CO}_{2}$ ) ou 0,03 p. 100 (lot témoin) de gaz carbonique et, après ces périodes, la répartition des électrolytes et du glucose libre entre l'albumen et le vitellus a été étudiée.

Le pH de l'albumen (fig. I-I) est constant dans le lot $\mathrm{CO}_{2}$ aux environs de 8,2 et atteint 9,3 chez les œufs témoins ; dans le premier cas, il subsiste en outre un gradient de pH d'une couche de l'albumen à la suivante, ce qui n'a pas lieu en l'absence de gaz carbonique. Réciproquement, la $\mathrm{COO}_{2}$ (fig. I-II) reste supérieure de I2 à $\mathrm{I} 5 \mathrm{~mm} \mathrm{Hg}$ dans l'albumen du lot $\mathrm{CO}_{2}$.

La pression osmotique du blanc (fig. 2) diminue également dans les conditions habituelles de stockage, mais reste constante en présence de gaz carbonique. Il en est de même des teneurs en $\mathrm{Ca}$ et $\mathrm{Mg}$ (fig. 4). Nous observons en outre un transfert d'eau du blanc vers le jaune au cours du vieillissement de l'œuf, transfert modéré par la présence de $\mathrm{CO}_{2}$ (fig. 3). Celui-ci n'exerce en revanche aucune action sur les teneurs en glucose libre de l'albumen et du vitellus qui diminuent en moyenne de 15 et 56 p. Ioo respectivement.

$\mathrm{La}$ concentration de $\mathrm{K}$ dans l'albumen reste constante et celle de $\mathrm{Na}$ diminue quel que soit le mode de conservation (fig. 5).

Ces résultats sont interprétés, d'une part en fonction du rôle du pH de l'albumen dans le contrôle de la membrane vitelline, d'autre part en supposant que des liaisons entre les cations divalents et les protéines gouverneraient en partie la pression osmotique de l'albumen.

\section{INTRODUCTION}

Au cours du vieillissement de l'œuf, on assiste à des modifications physico-chimiques de ses constituants qui s'accompagnent d'une diminution des qualités marchandes et d'anomalies du développement embryonnaire. Nous avons déjà eu l'occasion de publier une mise à jour bibliographique à ce sujet (SAUVEUR, I967). 
Parmi les études consacrées à l'altération des constituants de 1'œuf, celles relatives aux protéines sont de loin les plus nombreuses; elles ont été résumées par FEENEY et Hru, (I960) et ShENSTONE (I968). Une des plus récentes met bien en évidence les altérations spécifiques des résidus glucidiques de l'ovomucine (KATO, NAKAMURA et SaTo, I970).

Plus rares ont été les travaux concernant certaines enzymes (glycosidases, LuSH et ConchiE, I966), les vitamines (CALET et BLUM, I970) et les électrolytes de l'œuf (SATro et al., I960).

Par ailleurs, la viabilité des embryons à la suite d'un stockage est améliorée si les œufs sont entreposés dans une atmosphère faiblement enrichie en $\mathrm{CO}_{2}$ (SAUVEUR, Ferré et Lacassagne, I967 ; Spencer, PetTitione et Becker, Ig68). On ignore le mode d'action exact du mélange gazeux; on sait simplement qu'il confère aux conalbumines de l'albumen un comportement électrophorétique identique à celui de l'œuf frais (CroizIER et SAUVEUR, I967). De plus, les transferts d'eau de l'albumen vers le jaune sont limités si l'on évite l'élévation de $\mathrm{pH}$ de l'albumen et, indirectement, les changements de structure de la couche externe de la membrane vitelline (Fromm et Gammon, I968).

Cependant, compte tenu de l'importance du glucose libre (Romanoff, r960) et de la pression osmotique de l'œuf (Austic, Grau et Matteson, Ig66) dans l'initiation du développement embryonnaire, on peut se demander si ces paramètres sont ou non affectés par la conservation de l'œuf.

Pour répondre à cette question, nous avons mesuré le $\mathrm{pH}, 1 \mathrm{a} p \mathrm{CO}_{2}$, la pression osmotique, la teneur en électrolytes, en eau et en glucose de l'albumen et du jaune d'œuf conservés dans une atmosphère enrichie ou non avec 2 p. Ioo $\mathrm{CO}_{2}$. Nous rapportons ci-après les résultats de ces essais, nous réservant d'exposer dans une publication ultérieure les relations qui pourraient exister entre les modifications de ces paramètres et le développement embryonnaire.

\section{MATÉRIEL ET MÉTHODES}

Les œufs étudiés proviennent de trois poules de type Leghorn (souche L 22) ; ramassés chaque jour, ils sont placés alternativement pour chaque animal dans chacune des deux ambiances de conservation suivantes : l'une (lot " $\mathrm{CO}_{2}$ ) renferme 2 p. Ioo de gaz carbonique, à une température de $12^{\circ} \mathrm{C}$ et une humidité relative de $80 \mathrm{p}$. roo. Les caractéristiques de la seconde (lot témoin) sont identiques exception faite du $\mathrm{CO}_{2}$ dont le taux est celui de l'air normal $(0,03 \mathrm{p}$. roo). La teneur en $\mathrm{CO}_{2}$ du premier local est contrôlée automatiquement par un analyseur à infrarouge (Contrôle de chauffe. Licence Onera).

Deux œufs de chaque animal et de chaque lot sont analysés après un temps de conservation compris entre I-3, IO-I 3 et 20-2I jours. Nous disposons ainsi de 6 oufs ( $2 \times 3$ poules) par traitement et stade de conservation étudiés.

A la fin de chaque période, les œufs sont placés, à l'intérieur du local " $\mathrm{CO}_{2}$ ", dans un dessiccateur contenant le mélange gazeux puis transportés au laboratoire. Ils sont alors cassés et au moyen de quatre seringues on prélève immédiatement un échantillon de vitellus et un de chacune des trois zones de l'albumen (épaisse, liquides interne et externe). Dans un délai maximum de ro minutes, on mesure le $\mathrm{pH}$ (microélectrode Unit $\mathrm{E} 502 \mathrm{I}$ Radiometer) et la $p \mathrm{CO}_{2}$ (microtonomètre AMT I Radiometer) de l'albumen. La pression osmotique de $0,2 \mathrm{ml}$ de ces mêmes échantillons est mesurée par abaissement du point de congélation (Fiske Osmometer). On pèse, à o, I mg près, I $g$ environ du contenu des 4 seringues, utilisé, après dilution au $I / 50$, pour le dosage du glucose libre (microdosage non spécifique par réduction du ferricyanure de potassium à l'AutoAnalyseur Technicon). 
L'albumen et le jaune restants sont placés à l'étuve à I I $0^{\circ} \mathrm{C}$ pendant 24 heures avant détermination de la matière sèche puis calcinés au four à $400^{\circ} \mathrm{C}$ pendant 24 heures; les cendres sont reprises par $\mathrm{HNO}_{3} \mathrm{~N} / 5$. Na et $\mathrm{K}$ sont dosés par spectrophotométrie de flamme (Eppendorf), Ca et Mg par spectrophotométrie d'absorption atomique (Hilger et Watts) et $\mathrm{Cl}$ par potentiométrie au nitrate d'argent (électrode Radiometer P 40I). Des travaux antérieurs (SATo et al., I960; SAUVEUR, I969) ayant montré que ces éléments sont uniformément répartis dans l'albumen, nous ne distinguons pas les trois zones de celui-ci pour cette dernière partie de notre étude.

L'analyse statistique des résultats est conduite suivant des plans factoriels puisque chaque aninual est également représenté par deux œufs dans chacun des traitements. Nous pouvons étudier ainsi l'effet du mode de conservation ( 2 niveaux), de l'âge de l'œuf ( 3 niveaux), de chaque zone de l'albumen ( 3 niveaux) et éventuellement de chaque poule ( 3 niveaux) ainsi que les interactions entre ces divers paramètres.

\section{RÉSULTATS}

$$
\text { r. }-p \mathrm{H}-\mathrm{pCO}_{2} \text { de l'albumen }
$$

Les valeurs de $\mathrm{pH}$ et de $p \mathrm{CO}_{2}$ enregistrées autour des $2^{\mathrm{e}}$, $\mathrm{I} 2^{\mathrm{e}}$ et $2 \mathrm{O}^{\mathrm{e}}$ jours de conservation sont représentées par les figures I-I et I-II respectivement. Les résultats de 1'analyse de variance sont donnés au tableau $I$.

$$
2 \% \mathrm{CO}_{2} \quad \text { Témoins }
$$
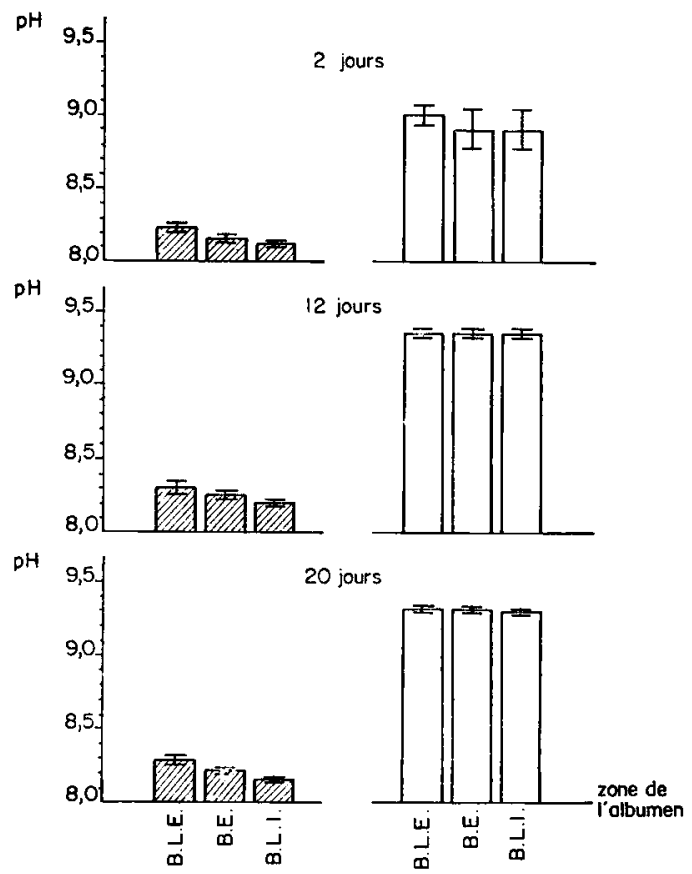

FIG. I. I. Evolution $d u p H$ de chaque zone de l'albumen * (signification statistique, voir tableau $I$ ).

B. L. E. = Blanc liquide externe

B. E. = Blanc épais

B. L. I. = Blanc liquide interne

* Sur toutes les figures, chaque valeur représente $\bar{x} \pm \frac{\sigma}{\sqrt{n}}$ de 6 mesures et on compare les effets de 2 et 0 p. roo de $\mathrm{CO}_{2}$ dans l'atmosphère de conservation de l'œuf. 
Le pH moyen de l'albumen des œufs conservés sous $2 \mathrm{p}$. Ioo $\mathrm{CO}_{2}$ n'évolue pas de façon significative au cours des trois semaines de conservation et se maintient aux environs de 8,2. A l'opposé, il est de 8,93 dans les œufs gardés 2 jours en atmosphère normale et augmente de façon significative jusqu'à 9,34 après I2 jours de stockage $(P<0,00 I)$; il est ensuite constant jusqu'à 20 jours dans ces mêmes œufs témoins. Le $\mathrm{CO}_{2}$ atmosphérique assure en outre la permanence d'une différence de $\mathrm{pH}$ entre les trois zones de l'albumen étudiées ; cette différence est de 0,05 unité entre chaque zone, quel que soit l'âge de l'œuf $(\mathrm{P}<0,05$ entre les deux zones liquides externe et interne). Ce gradient de $\mathrm{pH}$ à l'intérieur de l'albumen a, en revanche, totalement disparu chez les œufs conservés I2 jours sans $\mathrm{CO}_{2}$.

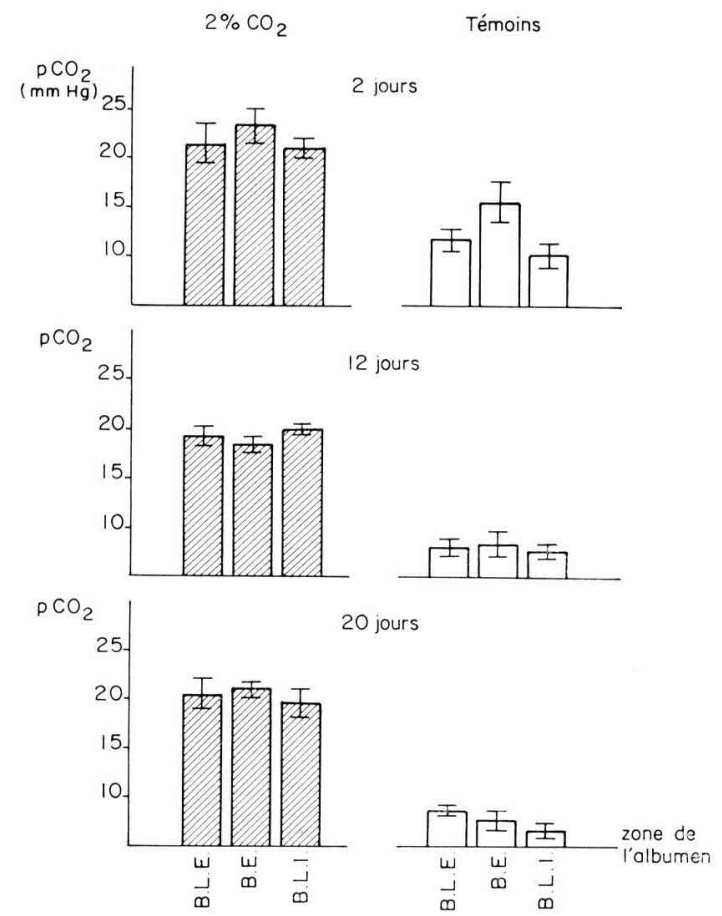

FIG. I. II. - Évolution de la $\mathrm{pCO}_{2}(\mathrm{~mm} \mathrm{Hg}$ ) de chaque zone de l'albumen

(signification statistique, voir tableau $\mathrm{I}$ )

B. L. E., B. E., B. L. I. : voir fig. I. I.

Les valeurs de pression partielle de $\mathrm{CO}_{2}$ dans l'albumen (fig. I-II) sont plus hétérogènes mais permettent cependant de constater que la $p \mathrm{CO}_{2}$ moyenne reste supérieure de I2 unités environ chez les œufs gardés sous 2 p. Ioo de $\mathrm{CO}_{2}$ comparés aux témoins $(\mathrm{P}<0,0005)$. Dans les deux lots les écarts entre zones disparaissent au cours du stockage; de plus les écarts-types calculés pour chaque zone diminuent en fonction du temps. Il y a donc homogénéisation progressive de la $p \mathrm{CO}_{2}$ entre les différentes zones de 1'albumen; la valeur finale, déjà établie à 12 jours, dépend de la concentration du $\mathrm{CO}_{2}$ dans l'atmosphère de stockage. Dans les deux cas étudiés ici, l'albumen liquide interne est la zone qui présente les tracés de décroissance les plus réguliers. 


\section{2. - Pression osmotique de l'albumen}

L'évolution de la pression osmotique des trois zones de l'albumen est représentée sur la figure 2 et son analyse de variance au tableau I. Nous observons qu'au-delà de $\mathrm{I} 2$ jours de conservation des oufs, le $\mathrm{CO}_{2}$ atmosphérique au taux de 2 p. Ioo assure le maintien d'une pression osmotique supérieure en moyenne de $20 \mathrm{mOsm} / 1$ environ à celle enregistrée dans l'albumen des œufs témoins $(P<0,0005)$.

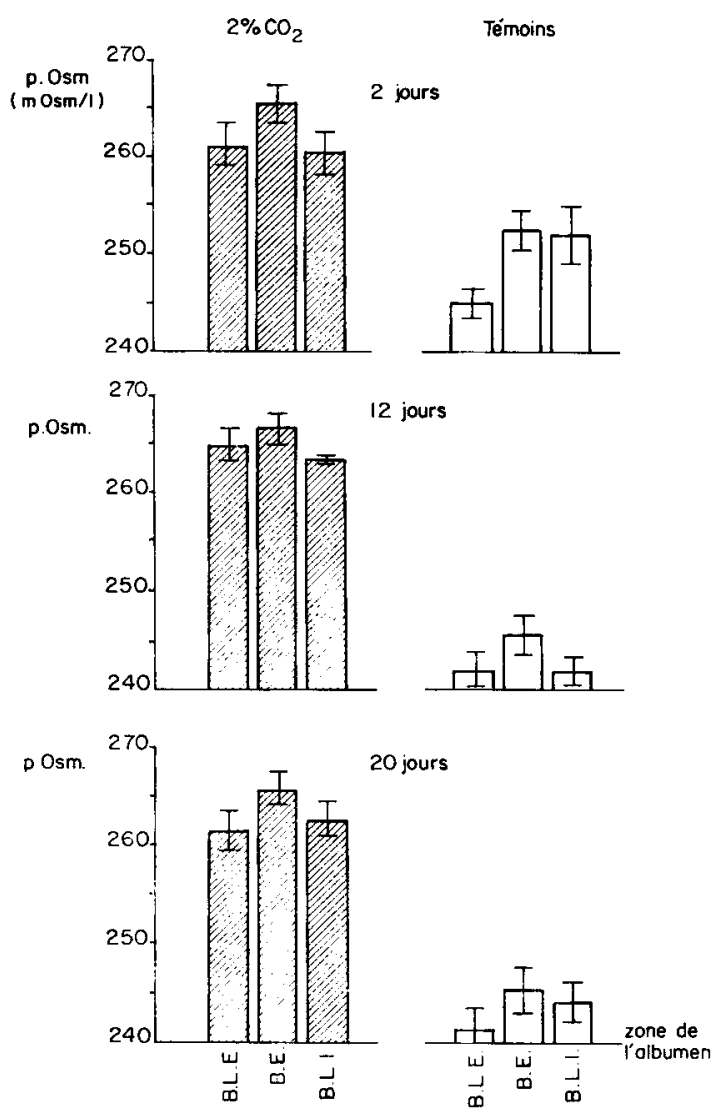

Fig. 2. - Evolution de la pression osmotique $(\mathrm{mOsm} / \mathrm{l})$ de chaque zone de l'albumen (signification statistique, voir tableau I).

B. L. E., B. E., B. L. I. : voir fig. I. I.

Comme nous l'avons noté pour les valeurs de $\mathrm{pH}$, celles de pression osmotique restent constantes à partir du deuxième stade d'observation pour les œufs témoins ; elles sont invariables pendant toute la durée du stockage pour les œufs conservés sous 2 p. $100 \mathrm{CO}_{2}$.

Il se maintient chez les deux lots une différence entre zones de l'albumen; la pression osmotique de l'albumen épais, calculée sur l'ensemble des données, est supérieure à celle des zones liquides externe et interne qui ne diffèrent pas entre elles. 
TABLEAU I

Analyse de variance des valeurs de $p \mathrm{H}, \mathrm{pCO}_{2}$ et pression osmotique de l'albumen et de la teneur en eau et en glucose de l'albumen et du jaune

(voir fig. I-I, I-II, 2 et 3 )

Valeurs de $\mathrm{F}$

\begin{tabular}{|c|c|c|c|c|c|c|c|}
\hline \multirow{2}{*}{ Effet mesuré } & \multicolumn{5}{|c|}{ Albumen } & \multicolumn{2}{|c|}{ Jaune } \\
\hline & $\mathrm{pH}$ & $p \mathrm{CO}_{2}$ & $\begin{array}{c}\text { Pression } \\
\text { osmotique }\end{array}$ & Eau & Glucose & Eau & Glucose \\
\hline Traitement $2 \%$ & & & & & & & \\
\hline $\mathrm{CO}_{2}$ vs $0 \% \ldots$ & $1526^{* * * *}$ & $347 * * *$ & $352 * * *$ & $4,5 *$ & 1,4 & 3,2 & - \\
\hline Age de l'œuf ... & $37 * * *$ & $14,4 * * *$ & 2,7 & $14,1 * * *$ & $30,2 * * *$ & $3,9 *$ & $5,7 *$ \\
\hline Poule $\ldots . . . \ldots$ & - & - & - & $142^{* * *}$ & - & - & - \\
\hline Zone de l'albu- & & & & & & & \\
\hline$\underset{\text { Traitement }}{\operatorname{men}} \ldots \ldots \ldots$ & $3,7^{*}$ & 2,1 & $6,6 * * *$ & - & - & - & 一 \\
\hline$\times$ âge $\ldots$ & $21^{* * *}$ & 2,7 & $9,5 * * *$ & - & - & - & 一 \\
\hline
\end{tabular}

$* p<0,05$

$* * p<0,01$

$* * * p<0.001$.

(Les données manquantes sont inférieures à 1.)

\section{3. - Eau et glucose de l'albumen et du jaune}

Les teneurs en eau et en glucose de l'albumen et du jaune sont rapportées à la figure 3 (analyse de variance au tableau I). Notons tout d'abord que la teneur en eau de l'albumen rapportée à la matière sèche, varie considérablement d'une poule à l'autre (tabl. $4 ; \mathrm{F}$ poule $=$ I42). Cet effet de l'animal est de loin la source de variation la plus importante. Par ailleurs, cette teneur en eau diminue entre le $2^{\mathrm{e}}$ et le

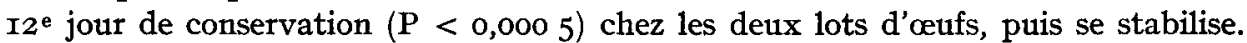
Ia baisse d'hydratation de l'albumen est cependant moins marquée dans les oufs gardés sous 2 p. Ioo $\mathrm{CO}_{2}$ que dans ceux du lot témoin $(\mathrm{P}<0,05)$. Le $\mathrm{CO}_{2}$ ambiant exercerait donc une action soit sur l'évaporation soit sur les transferts d'eau à l'intérieur de l'œuf.

La deuxième hypothèse semble la plus probable puisque l'hydratation dujaune des œufs conservés 20 jours sous $2 \mathrm{p}$. Ioo $\mathrm{CO}_{2}$ semble réduite par rapport à celle des cufs témoins. Cependant la différence entre les deux moyennes n'atteint pas le seuil de signification $5 \mathrm{p}$. Ioo du fait de la variabilité importante des mesures à l'intérieur de chacun des traitements. En revanche, l'accroissement moyen inter-traitement de la teneur en eau du jaune entre le $\mathrm{I}^{\mathrm{e}}$ et $1 \mathrm{e} 2 \mathrm{O}^{\mathrm{e}}$ jour de conservation est significatif $(\mathbf{P}<0,05)$. Il se produit donc de façon certaine un transfert d'eau de l'albumen vers le jaune, transfert qui paraît réduit par la présence de $\mathrm{CO}_{2}$.

Les teneurs en glucose (fig. 3 ) de l'albumen et du jaune apparaissent absolument indépendantes de la présence de $\mathrm{CO}_{2}$ dans l'atmosphère de conservation. De plus, l'analyse de variance montre que cet élément est réparti uniformément entre les trois zones de l'albumen ( $F$ entre zones $<I$ ) : c'est pourquoi nous n'avons pas fait figurer 
sur la figure 3 les valeurs individuelles de chaque zone. L'âge de l'œuf exerce un effet significatif sur la teneur en glucose de l'albumen et du jaune : le contenu de l'albumen reste stable entre 2 et $\mathrm{I} 2$ jours puis diminue de $I 5$ p. roo entre $I 2$ et 20 jours de conservation. A l'opposé, la concentration enregistrée dans le jaune diminue entre 2 et I 2 jours puis n'évolue plus ; la baisse enregistrée sur l'ensemble des trois semaines et des deux traitements représente ainsi 56 p. roo de la teneur initiale du jaune en glucose ; seule une faible part peut être expliquée par l'accroissement d'hydratation de ce milieu.
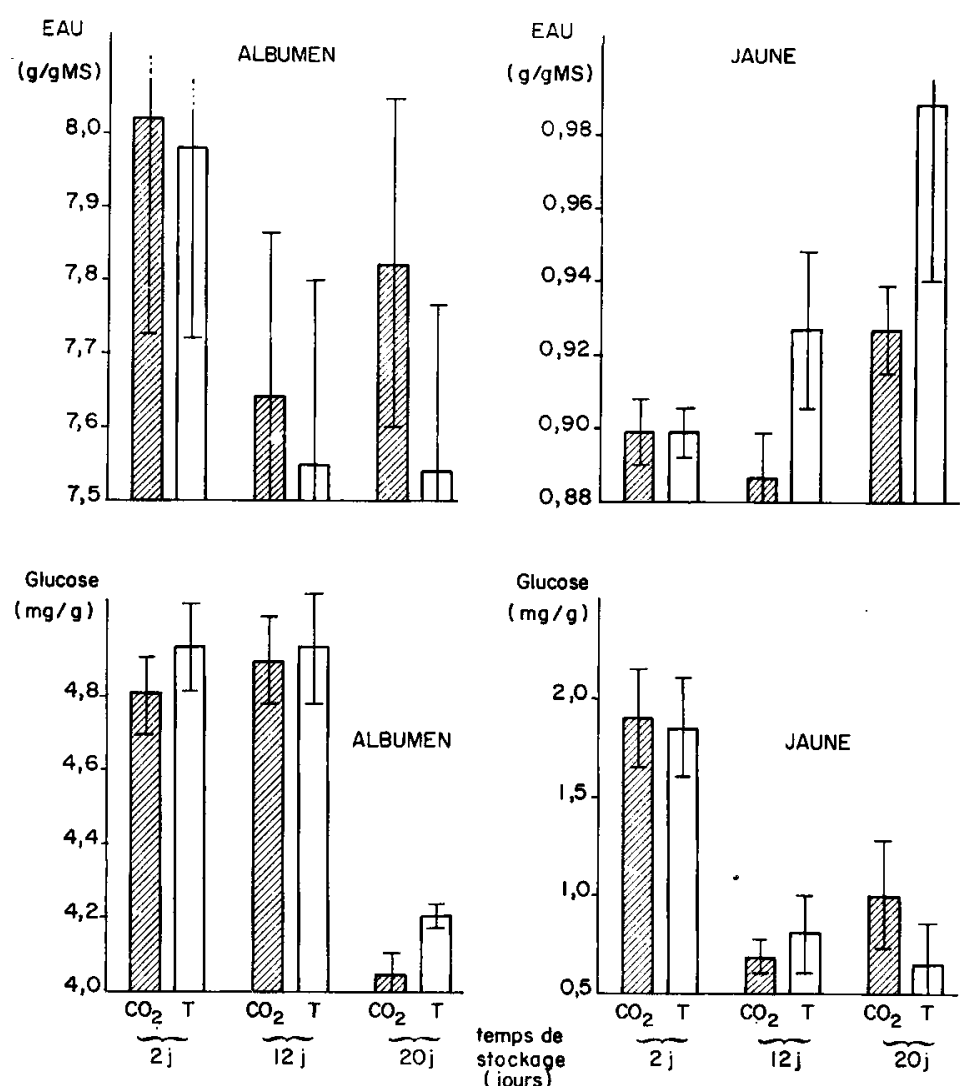

Fig. 3. - Teneurs de l'albumen et du jaune en eau (g/g de matière sèche) et en glucose libre (g/g de poids frais) (signification statistique, voir tableau $\mathrm{I}$ )

En résumé, si l'on estime à 30 et $20 \mathrm{~g}$ les poids respectifs de l'albumen et du jaune, on s'aperçoit que 1'œuf total perd en trois semaines de conservation, $44 \mathrm{mg}$ de glucose environ sur les $184 \mathrm{mg} q \mathrm{qu}^{\prime}$ il contenait initialement, soit $24 \mathrm{p}$. Ioo.

\section{4. - Cations divalents de l'albumen et du jaune}

Comme nous l'avons dit précédemment, il n'a pas été tenu compte pour tous les éléments suivants des différentes zones de l'albumen.

Le tableau 2 donne les teneurs de l'albumen et du jaune en calcium et magné- 


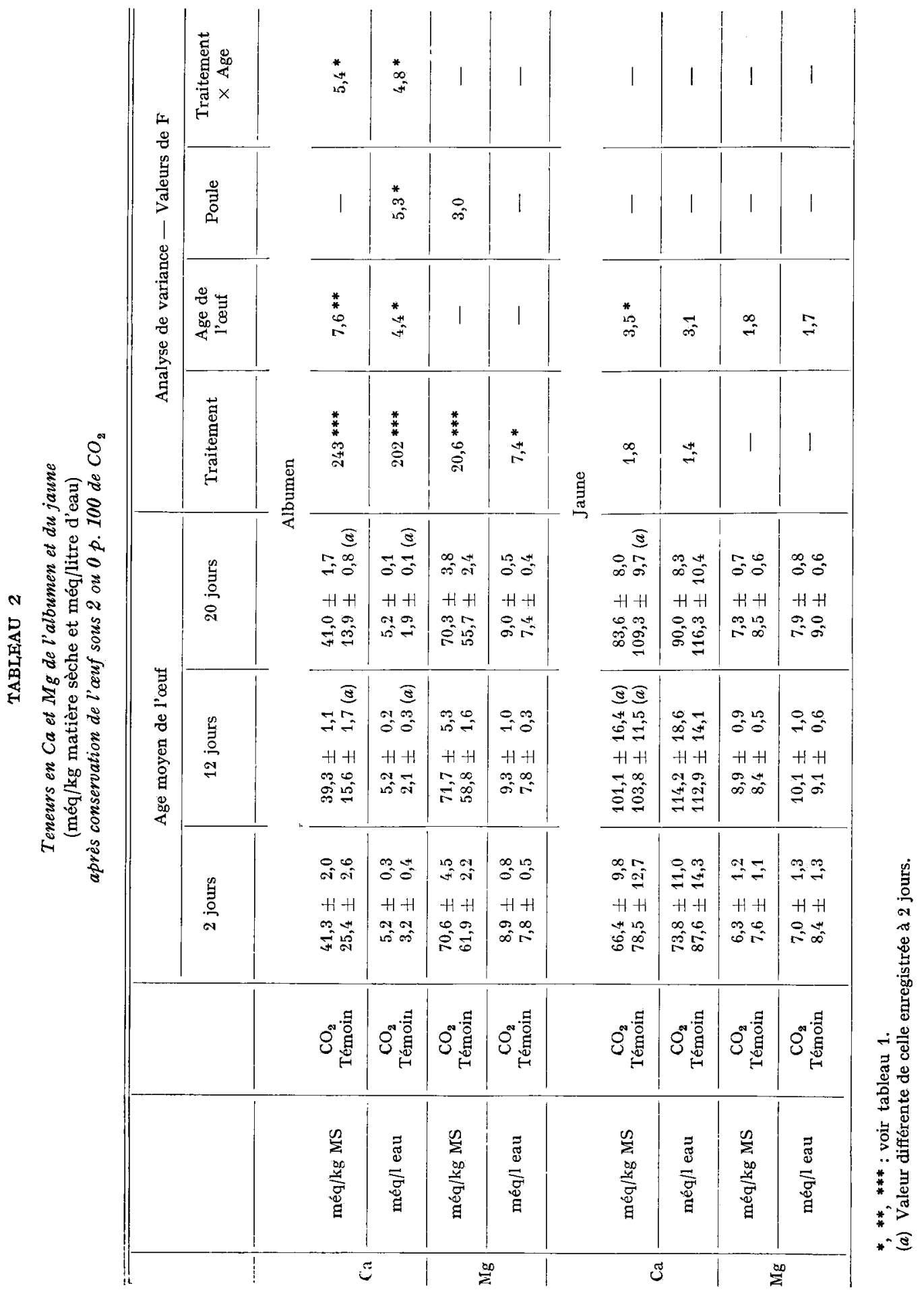


sium rapportées à la matière sèche et à l'eau présente, ainsi que les principaux résultats de l'analyse de variance. Pour plus de clarté, les valeurs relatives à la matière sèche de l'albumen, qui sont les plus intéressantes, sont également illustrées par la figure 4 .

\section{a) Calcium.}

La teneur en $\mathrm{Ca}$ de l'albumen des œufs témoins rapportée à la matière sèche, diminue de 25 à 15 méq $/ \mathrm{kg}$ entre 2 et $\mathrm{I} 2$ jours de conservation $(\mathrm{P}<0,05)$ puis se stabilise. A l'opposé, elle est parfaitement stable chez les œufs conservés sous $\mathrm{CO}_{2}$ et les valeurs enregistrées lors du I ${ }^{\text {er }}$ stade d'observation (âge moyen de l'œuf : 2 jours) diffèrent déjà au profit du lot $\mathrm{CO}_{2}$. Le $\mathrm{CO}_{2}$ atmosphérique exerce donc une action stabilisatrice importante sur le calcium de l'albumen; elle se traduit sur la moyenne des trois stades étudiés par les deux valeurs :

$$
\begin{aligned}
& \bar{x}_{\mathrm{CO}_{2}}=40,5 \text { méq. } \mathrm{Ca} / \mathrm{kg} \text { matière sèche } \\
& \bar{x}_{\text {Tumoin }}=\mathrm{I} 8,3 \text { méq. } \mathrm{Ca} / \mathrm{kg} \text { matière sèche }
\end{aligned}
$$

Nous aboutissons aux mêmes conclusions en considérant les teneurs en $\mathrm{Ca}$ de l'albumen rapportées à l'eau présente (tabl. 2). L'effet du $\mathrm{CO}_{2}$ atmosphérique sur la concentration du $\mathrm{Ca} /$ eau de l'albumen reste très élevé bien que tempéré par l'action freinatrice de ce gaz sur la perte d'eau de l'albumen (voir plus haut). Il s'y ajoute un " effet poule " significatif (tabl. 2) qui concorde avec celui enregistré sur la teneur en eau (tabl. I) ; la plus faible valeur de $\mathrm{Ca}$ /eau observée systématiquement dans les œufs d'une des poules n'est donc due qu'à une plus grande dilution.

Les valeurs enregistrées dans le jaune (tabl. 2) sont surtout marquées par une extrême variabilité à l'intérieur des traitements qui rend très difficile la mise en évidence de leurs effets. L'accroissement, en fonction du temps, du contenu en calcium du jaune rapporté à la matière sèche atteint toutefois le seuil de signification sur l'ensemble des deux lots. Il semble de plus que le $\mathrm{CO}_{2}$ modère cette augmentation, surtout lorsque l'œuf est conservé durant 20 jours ; il n'est pas possible de conclure statistiquement. Compte tenu de la grande disproportion entre les teneurs en Ca de 1'albumen et du jaune (20 fois plus de $\mathrm{Ca}$ dans le jaune, calculé par rapport à l'eau et ro fois plus par rapport au poids frais), il est cependant normal que l'action stabilisatrice du $\mathrm{CO}_{2}$ sur le $\mathrm{Ca}$ de l'albumen n'apparaisse que très faiblement au niveau du jaune.

b) Magnésium.

Les effets de la conservation sur la teneur en magnésium de l'albumen (rapportée à la matière sèche) apparaissent à peu près identiques à ceux observés pour le calcium avec une amplitude moindre. Le contenu en $\mathrm{Mg}$ de l'albumen des œufs témoins diminue en effet progressivement alors qu'il est parfaitement stable dans les œufs conservés sous $\mathrm{CO}_{2}$ (cf. tabl. 2 et fig. 4). L'effet de ce traitement apparaît très hautement significatif $(\mathbf{P}<0,0005)$ sur l'ensemble des trois périodes étudiées. Cet effet stabilisateur du $\mathrm{CO}_{2}$ sur le contenu en $\mathrm{Mg}$ de l'albumen se retrouve en considérant les concentrations de $\mathrm{Mg}$ par rapport à l'eau ; comme précédemment, il est cependant moins net $\mathrm{du}$ fait de la moindre perte d'eau de l'albumen sous $\mathrm{CO}_{2}$.

Au niveau du jaune, la dispersion des valeurs de $M g$ est telle qu'il n'apparaît aucun effet significatif du $\mathrm{CO}_{2}$ atmosphérique ou du temps de conservation. 

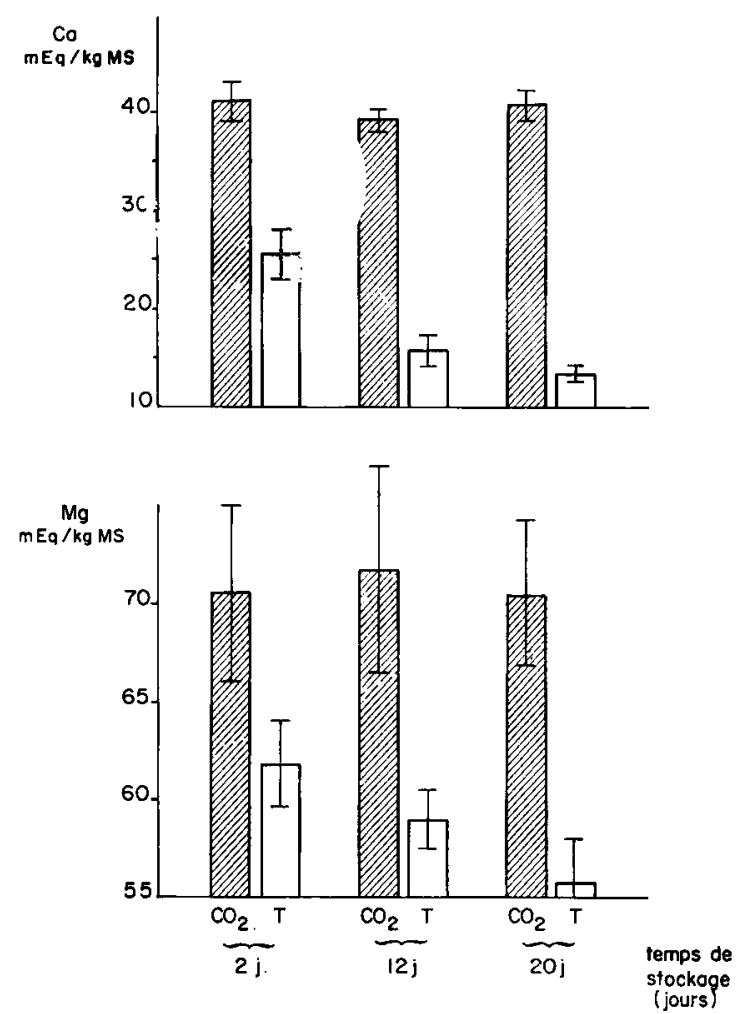

İic. 4. - Teneurs en calcium et magnésium (méq/kg de matière sèche) de l'albumen (signification statistique, voir tableau 2)

\section{5. - Ions monovalents de l'albumen et du jaune}

a) Sodium.

Nous donnons au tableau 3 les moyennes des teneurs en sodium et potassium de l'albumen et du jaune rapportées à la matière sèche et à l'eau du milieu. Comme ci-dessus, la figure 5 est consacrée spécialement aux teneurs de l'albumen rapportées à la matière sèche.

Le contenu en Na de l'albumen varie beaucoup d'une poule à l'autre $(P<0,0005$, tabl. 3) : le classement des 3 poules étudiées est d'ailleurs le même que celui observé pour la teneur de l'albumen en eau comme l'indique le tableau 4. Ce contenu varie également avec l'âge de l'œuf : il diminue surtout entre 2 et $\mathrm{I} 2$ jours $(\mathrm{P}<0, \mathrm{OI})$ puis se stabilise. Le $\mathrm{CO}_{2}$ atmosphérique ne semble pas freiner cette baisse du contenu sodé de l'albumen contrairement à ce que nous avons décrit pour Ca et $\mathrm{Mg}$.

$\mathrm{Si}$ nous considérons les concentrations de $\mathrm{Na}$ dans l'eau (tab1. 3) seul " l'effet poule " reste significatif, celui du temps de conservation n'agissant plus. Ceci est en accord avec le diminution du contenu en eau représentée sur la figure 3. C'est donc 1a quantité totale de sodium de l'albumen qui est affectée par la conservation de l'œuf, sa concentration dans l'eau du milieu restant constante. Au niveau du jaune, 


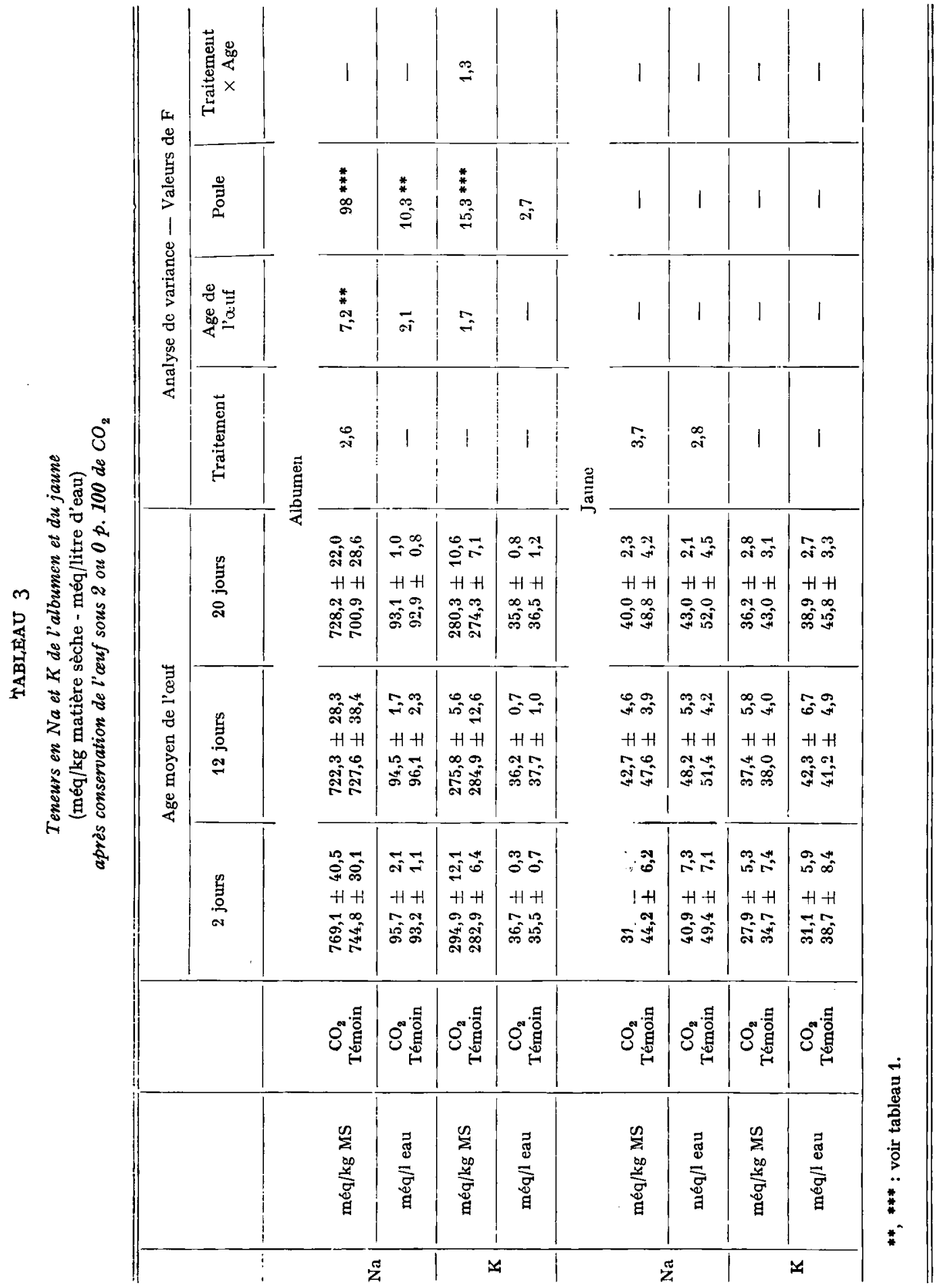


aucun des paramètres étudiés n'affecte significativement la concentration du sodium ; celle-ci semblerait être réduite par la présence de $\mathrm{CO}_{2}$ dans l'atmosphère de conservation, mais cet effet éventuel est masqué par la dispersion des données.
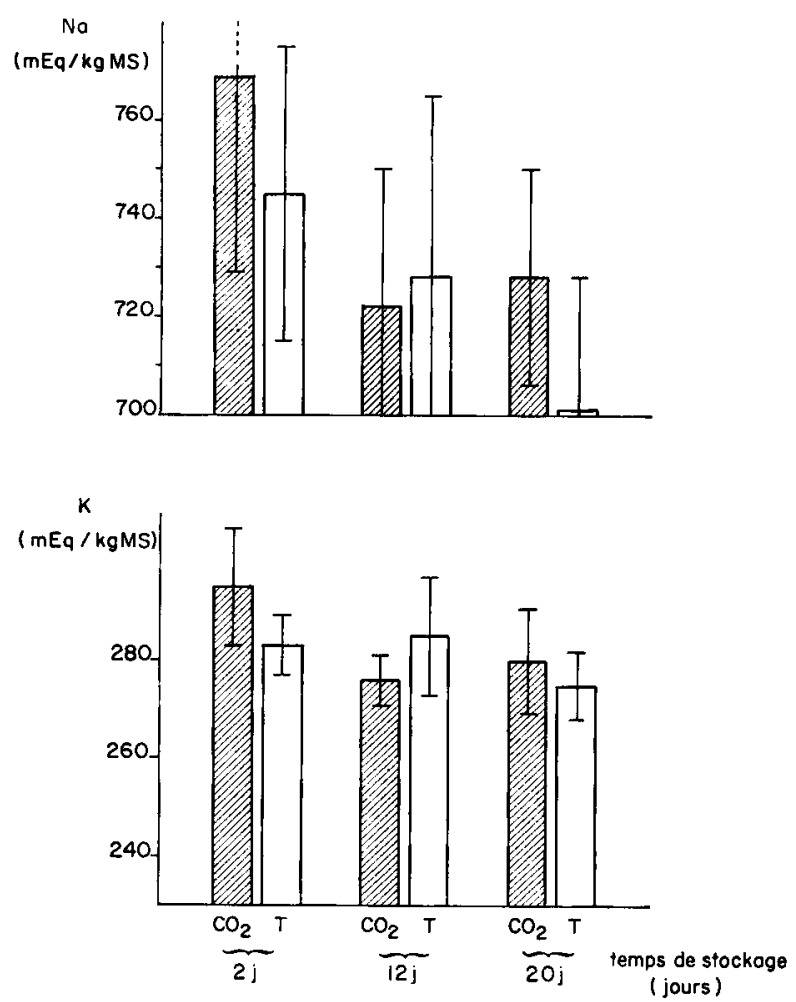

FIG. 5. - Teneurs en sodium et potassium (méq/kg de matière sèche) de l'albumen (signification statistique, voir tableau 3 )

b) Potassium (tabl. 3).

Nous enregistrons comme ci-dessus un effet de l'animal sur le contenu en $K$ de l'albumen ( $\mathrm{P}<0,0005)$ qui évolue dans le même sens que $\mathrm{Na}$ et l'eau (voir tabl. 4). Cette fluctuation individuelle disparaît lorsque $\mathrm{K}$ est rapporté à l'eau du milieu. Par ailleurs, le potassium semble être l'élément dont la concentration reste la plus constante au cours de la conservation de l'œuf, tant dans l'albumen que dans le jaune. Ni l'âge de l'œuf, ni le $\mathrm{CO}_{2}$ de l'atmosphère n'affectent significativement ce paramètre.

c) Chlore.

Les teneurs de l'albumen en chlore sont représentées par la figure 6; celles du jaune n'ont pas été enregistrées. L'analyse de variance de la teneur en $\mathrm{Cl}$ rapportée à la matière sèche de l'albumen montre un effet de la poule $(P<0,0005)$ identique à celui enregistré pour l'eau, $\mathrm{K}$ et $\mathrm{Na}$ (tabl. 4). Comme dans le cas du potassium, cet effet disparaît si l'on considère la concentration de $\mathrm{Cl}$ dans l'eau de l'albumen. 
Le temps et le mode de conservation de l'œuf (fig. 6) n'affectent pas le contenu total en $\mathrm{Cl}$ de l'albumen mais il apparaît une légère augmentation de la concentration dans l'eau de ce milieu $(P<0,05)$ entre le $2^{\mathrm{e}}$ et le $\mathrm{I} 2^{\mathrm{e}}$ jour de stockage; les valeurs restent ensuite inchangées. Il est donc clair que cet accroissement de $\mathrm{Cl} /$ eau n'est dû qu'à la diminution du contenu en eau de l'albumen (fig. 3). La présence de $\mathrm{CO}_{2}$ semble d'ailleurs freiner le phénomène mais il n'est pas possible de trancher statistiquement $(\mathrm{F}=3,96$ contre $4,4 \mathrm{I}$ à $\mathrm{P}=0,05)$.
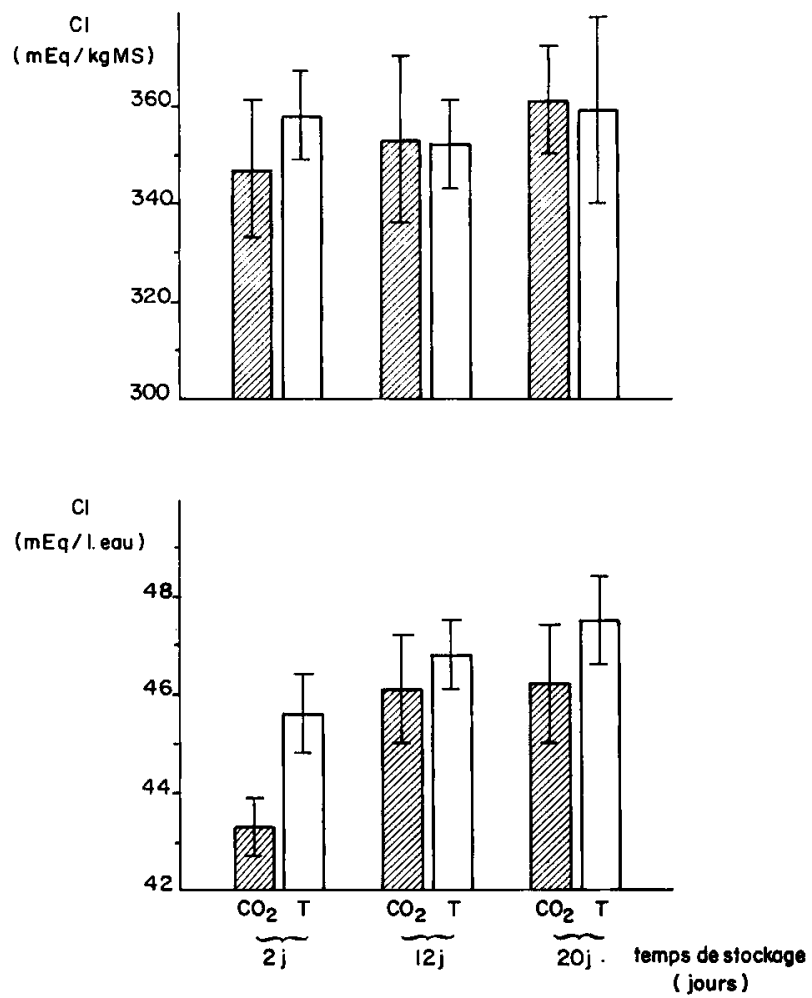

Fı́. 6. - Teneur en chlore de l'albumen (méq/kg de matière sèche et méq/l d'eau)

\section{TABLEAU 4}

Moyennes par poule des teneurs de l'albumen en eau, $\mathrm{Na}^{+}, \mathrm{K}^{+}$et $\mathrm{Cl}^{-}$

\begin{tabular}{c|c|c|c|c}
\hline \hline Poule & $\begin{array}{c}\text { Eau } \\
\text { (g/g MS) }\end{array}$ & $\begin{array}{c}\mathrm{Na}^{+} \\
\text {(méq/kg MS) }\end{array}$ & $\begin{array}{c}\mathrm{K}+ \\
\text { (méq/kg MS) }\end{array}$ & $\begin{array}{c}\mathrm{Cl}^{-} \\
\text {(méq/kg MS) }\end{array}$ \\
\hline & & 649,8 & 262,0 & \\
2 & 6,997 & 805,7 & 297,4 & 317,5 \\
3 & 8,299 & 746,0 & 287,2 & 375,3 \\
\hline
\end{tabular}




\section{DISCUSSION}

Nous avons conservé des oufs à couver de 2 à 2 I jours après la ponte dans des atmosphères contenant 2 ou o p. Ioo de $\mathrm{CO}_{2}$. Résumées, très rapidement, les actions du $\mathrm{CO}_{2}$ sur 1'équilibre hydro-minéral de l'albumen sont les suivantes :

- il maintient un gradient de $\mathrm{pH}$ dans l'albumen à un niveau moyen inférieur de I unité à celui observé chez les œufs témoins. Réciproquement, la $p \mathrm{CO}_{2}$ de ce milieu reste supérieure de $5 \mathrm{~mm} \mathrm{Hg}$;

- la pression osmotique de l'albumen reste constante en présence de $\mathrm{CO}_{2}$ mais diminue de $20 \mathrm{mOsm} / 1 \mathrm{chez}$ les cufs témoins. De même le gaz carbonique assure la permanence de la teneur en $\mathrm{Ca}$ et $\mathrm{Mg}$ de l'albumen et semble freiner leur transfert vers le jaune. Il ralentirait également, les transferts d'eau;

- à l'opposé, le gaz carbonique ne contrôle pas la teneur en sodium de l'albumen; de plus les contenus en glucose de l'albumen et du jaune diminuent de la même façon chez les œufs $\mathrm{CO}_{2}$ et témoins.

Ces résultats peuvent être discutés sous de multiples aspects tant sontcomplexes les équilibres existant à l'intérieur de l'œuf après la ponte. Nous allons essayer d'en aborder quelques-uns.

\section{I. - Échanges albumen-jaune; rôle du pH de l'albumen}

De nombreux travaux, résumés par Shenstone (I968) et CALET et BLUM (I970) dans leurs revues bibliographiques, ont montré que la membrane vitelline ne contrôle plus les transferts de l'albumen vers le vitellus après un certain temps de conservation de l'œuf. On sait de plus que l'accroissement de la teneur en eau du jaune n'est probablement pas dû̀ à un phénomène osmotique. Le terme même de membrane vitelline correspond à des notions périmées ; les études récentes y distinguent plusieurs couches d'origine et de nature fort différentes dont le rôle de " membrane " est fort discutable (Bel, AIrs, Harkness et Harkness, I963 ; BaIn et Hall, I969 ; Jensen, I969).

En ce qui concerne les éléments qui nous intéressent ici, à savoir les électrolytes, le seul travail que nous connaissons est celui de SATo et al. (Ig60) qui ont observé que, lors d'un stockage en atmosphère normale, la teneur de 1'albumen en $\mathrm{Na}, \mathrm{Ca}, \mathrm{Mg}$ et $\mathrm{CO}_{2}$ diminue alors que celle en $\mathrm{K}$ et $\mathrm{Cl}$ reste constante. Nos résultats (fig. 4, 5 et 6) sont en accord avec ceux de ces auteurs comme le montrent les pertes moyennes en pourcentage observées dans l'albumen en 20 jours de conservation (calculées à partir des variations de quantité totale de chaque élément pour SATo et al., et à partir des teneurs rapportées à la matière sèche dans notre cas).

\begin{tabular}{|c|c|c|c|c|}
\hline & $\mathrm{Ca}$ & $\mathrm{Mg}$ & $\mathrm{Na}$ & K \\
\hline at al & 一 & - & $一$ & - \\
\hline $\begin{array}{l}\text { SATO et al., } \\
\text { Résultats présents (1) }\end{array}$ & $\begin{array}{l}-47 \text { p. I00 } \\
-66\end{array}$ & $\begin{array}{l}-25 \\
-2 I\end{array}$ & $\begin{array}{l}-7 \\
-8.8\end{array}$ & $\begin{array}{l}0 \\
0\end{array}$ \\
\hline
\end{tabular}

Il est donc certain que, dans des conditions normales de conservation, il se produit de l'albumen vers le jaune, non seulement un mouvement d'eau, mais aussi un

(1) Variations calculées entre les moyennes du lot $\mathrm{CO}_{2}$ à 2 jours et celles du lot témoin à 20 jours. 
transfert d'ions. De ce point de vue, l'effet protecteur d'une ambiance enrichie en $\mathrm{CO}_{2}$ que nous démontrons ici nous semble intéressant. Il est connu depuis longtemps que le $\mathrm{CO}_{2}$ freine les transferts d'eau de l'albumen vers le vitellus (SMITH, I93 ; MORAN, I937). MORAN démontre même que la teneur de 2,5 p. IOO $\mathrm{CO}_{2}$ est suffisante et que l'emploi de $\mathrm{CO}_{2}$ pur n'exerce aucun effet bénéfique supplémentaire sur 1'index de forme de jaune ou sa teneur en eau.

Plus récemment, les travaux de Frovm (I967) et Fromm et Gammon (I968) ont montré que le $\mathrm{pH}$ de l'albumen conditionne les transferts d'eau à l'intérieur de l'œuf en contrôlant la structure fibrillaire de la couche externe de la "membrane " vitelline, apparentée aux chalazes. Ceci explique l'effet de $\mathrm{CO}_{2}$ ambiant puisque le pH de l'albumen dépend essentiellement de la teneur en $\mathrm{CO}_{2}$ de l'air ambiant (Brooks et PACE, I938) ; nous retrouvons évidemment ce résultat ici (fig. I-I). Nous montrons également que la présence de $2 \mathrm{p}$. Ioo $\mathrm{CO}_{2}$ dans 1'air supprime totalement les transferts très importants de calcium et de magnésium qui interviennent en atmosphère normale (fig. 4 et 5 ) et maintient une pression osmotique plus élevée dans l'albumen (fig. 2). Ce traitement est, en revanche, sans action significative sur les échanges de sodium.

Nous pouvons donc émettre l'hypothèse suivant laquelle non seulement les transferts d'eau, mais peut-être aussi ceux des cations divalents sont contrôlés par la couche externe gélifiée qui entoure le jaune de l'œuf frais.

\section{2. - Caractéristiques des trois zones de l'albumen}

Nous avons montré précédemment (SAUVEUR, I969) qu'il existe dans l'albumen de l'œuf, aussitôt après la ponte, un gradient de $\mathrm{pH}$, de $p \mathrm{CO}_{2}$ et de concentration du phosphore total : le pH augmente régulièrement depuis la zone fluide interne jusqu'à la zone fluide externe alors que $1 \mathrm{a} p \mathrm{CO}_{2}$ et la teneur en phosphore diminuent. A l'opposé, nous avons trouvé que les ions $\mathrm{Na}, \mathrm{K}$ et $\mathrm{Cl}$ sont uniformément répartis. SATo et al. (I960) rapportent une même égalité de répartition au cours de la conservation, fait qui nous a d'ailleurs conduit à ne pas séparer les différentes zones de l'albumen pour le dosage de ces éléments lors de la présente expérience. En revanche, nous observons (fig. I) que la présence de 2 p. Ioo de $\mathrm{CO}_{2}$ maintient des différences de $\mathrm{pH}$ significatives entre les 3 zones de l'albumen ; il n'en est pas de même pour la $p \mathrm{CO}_{2}$ qui devient rapidement uniforme (fig. 2) à l'intérieur de l'albumen.

Notons enfin que 1'albumen épais se caractérise par une pression osmotique légèrement supérieure à celle des zones liquides interne et externe, quels que soient apparemment le temps et le mode de conservation de l'œuf.

\section{3. - Relation pH-pression osmotique-cations divalents de l'albumen}

Les variations de pression osmotique de l'albumen, en fonction du temps et de 1'atmosphère de conservation, ne peuvent être liées ni au glucose ni aux cations monovalents (fig. 2) ; en effet, les concentrations de ces éléments sont, soit constantes (K), soit variables de la même façon chez les œufs gardés sous o ou 2 p. $100 \mathrm{CO}_{2}$ (glucose, $\mathrm{Na}$ et $\mathrm{Cl}$ ). Au contraire, il semble exister un rapport entre la pression osmotique et la teneur en cations divalents de l'albumen. Pour préciser ce fait, nous avons calculé sur l'ensemble des données disponibles les coefficients de corrélation entre la pression 
osmotique (mOsm/1) et les teneurs en calcium et magnésium de l'albumen (méq/kg matière sèche). Nous obtenons :

$$
\begin{aligned}
& r_{p \mathrm{O} \mathrm{m},}(\mathbf{c a})=0,89\left(\mathrm{~F}=\mathrm{I}_{3} 6 ; \mathrm{P}<0,0005\right) \\
& r_{p \text { 03 }},(\mathrm{Mg})=0,62(\mathrm{~F}=22 ; \mathrm{P}<0,0005)
\end{aligned}
$$

Ces relations peuvent être expliquées en partie par le fait que du calcium et, à un moindre degré, du magnésium semblent être transférés de l'albumen vers le jaune chez les témoins. Cependant, si nous considérons les concentrations de $\mathrm{Ca}$ et $\mathrm{Mg}$ par rapport à l'eau de l'albumen (tabl. 2) nous constatons que les écarts entre les œufs gardés sous $\mathrm{CO}_{2}$ et les témoins ne sont au maximum que de 3,3 et I,6 méq/1 respectivement soit au total $2,5 \mathrm{mM} / 1$. En ađmettant que cette quantité représente en totalité des ions en solution (ce qui n'est probablement pas le cas), la différence de pression osmotique observée entre les 2 lots ne devrait être que de $2,5 \mathrm{mOsm} / 1$; or, elle atteint près de $20 \mathrm{mOsm} / 1$ (fig. 2) au profit du lot $\mathrm{CO}_{2}$ chez lequel la teneur en eau de l'albumen reste par ailleurs plus élevée.

On peut alors faire intervenir les variations de $\mathrm{pH}$ de l'albumen. Un calcul de corrélation effectué (sur l'ensemble des 3 périodes de conservation) entre la pression osmotique et le $\mathrm{pH}$ de chaque zone de l'albumen donne les résultats suivants :

A1. liq. ext. : $p_{\mathrm{osm}}=-\mathrm{I} 8,95 \mathrm{pH}+4 \mathrm{I} 8,5$ avec $r=-0,88(\mathrm{~F}=\mathrm{II} 3, \mathrm{P}<0,0005)$ Al. ép. $\quad:-=-\mathrm{I} 7,44 \mathrm{pH}+408,5$ avec $r=-0,89(\mathrm{~F}=\mathrm{I} 24, \mathrm{P}<0,0005)$ Al. liq. int. : $-=-\mathrm{I}_{5}, 85 \mathrm{pH}+39 \mathrm{I}, 46$ avec $r=-0,89(\mathrm{~F}=\mathrm{I} 28, \mathrm{P}<0,0005)$

Les valeurs des trois coefficients de corrélation sont élevées et identiques; de plus, les variances des 3 coefficients de régression sont homogènes (test de Barlett) ; ceci nous autorise à comparer entre elles les pentes de chacune des 3 droites de régression qui apparaissent différentes de façon très hautement significative $\left(F_{2,102}\right.$ $=33, \mathrm{P}<0,0005)$.

I1. existe donc une forte liaison inverse entre $\mathrm{pH}$ et pression osmotique de l'albumen, liaison dont la pente varie régulièrement à l'intérieur de l'albumen. Cette relation, rapprochée de celles calculées plus haut entre la pression osmotique d'une part et les teneurs en $\mathrm{Ca}$ et $\mathrm{Mg}$ d'autre part, permet d'émettre 1'hypothèse suivante sur le rôle du pH. Lorsque le pH s'accroît (cas des œufs conservés à l'air) il s'éloigne du point isoélectrique des protéines de l'albumen, exception faite de celui du lyzozyme fortement basique (COTTERIL, et WINTER, I965) ; ces protéines présentent alors des charges négatives libres en nombre croissant susceptibles de fixer des cations inorganiques. On sait en particulier (WIILIAMS, I967; YoN, I969) que la stabilité des complexes formés avec les groupements $\mathrm{R}-\mathrm{COO}^{-}$, varie suivant la place du cation dans la série lyotropique de HoFMEISTER (I888) : $\mathrm{Mg}>\mathrm{Ca}>\ldots \mathrm{Na}>\mathrm{K}>\ldots$ Les cations divalents seraient donc préférentiellement fixés et pourraient former, entre les chaînes protidiques, des ponts électrostatiques responsables, en partie, de la diminution du pouvoir de rétention d'eau de 1'albumen épais, selon le schéma décrit par HAMm (I963) pour l'actomyosine. Le même phénomène a d'ailleurs été évoqué dans le cas du plasma pour expliquer la diminution relative du Ca ultrafiltrable liée à une augmentation du $\mathrm{pH}$ ('TEREPKA et al., I970).

Si une telle fixation existe dans l'albumen de l'œuf, elle entraîne une diminution de la concentration ionique et, par conséquent, de la pression osmotique totale de la solution protéique (GRÉMY et PAGES, I962). Cette hypothèse est soutenue par 1'obser- 
vation de SATo et al. (I960) qui ont noté que la portion dialysable des ions de l'albumen reste constante pendant la conservation, sauf dans le cas du calcium : elle représenterait $20 \mathrm{p}$. Ioo du $\mathrm{Ca}$ total de l'albumen en début de conservation, Io $\mathrm{p}$. Ioo après 6 jours, puis resterait constante. Un tel comportement temporel reflète bien celui observé ici pour la pression osmotique.

Cette hypothèse n'explique pas, en revanche, que les coefficients de régression liant $\mathrm{pH}$ et $p$ Osm aient une valeur différente dans les trois zones de l'albumen. Il existe en effet un gradient protéique croissant de l'extérieur à l'intérieur de l'albumen (Lush et CoNchIE, I966) ; suivant le raisonnement ci-dessus, la zone la plus riche en protéines (zone liquide interne) devrait être celle où une même élévation de $\mathrm{pH}$ affecte le plus la pression osmotique, ce qui n'est pas. Rappelons cependant que la fraction inorganique de l'albumen n'est pas la seule responsable de la pression osmotique de ce milieu et que la conservation de l'œuf affecte la répartition interne des protéines comme l'ont montré SaTo et Nakamura (I959) et KaTo, Nakamura et SaTo (I970).

\section{CONCLUSION}

Il se produit au cours de la conservation de l'œuf d'importantes modifications dans la répartition de l'eau et des électrolytes entre 1'albumen et le vitellus; ces modifications peuvent être modérées ou même supprimées en réduisant l'élévation de $\mathrm{pH}$ de l'albumen. Il semble que la pression osmotique de l'albumen soit également contrôlée par le $\mathrm{pH}$ du milieu; nous émettons l'hypothèse selon laquelle ce contrôle serait effectué par l'intermédiaire de liaisons entre les cations divalents et les charges négatives des protéines. Des travaux sont actuellement en cours pour vérifier si les faits décrits ici sont, ou non, en rapport avec les défauts de développement embryonnaire consécutifs à la conservation de 1'œuf.

Reçu pour publication en mai 1971.

\section{SUMMARY}

\section{GLUCOSE AND ELECTROLYTES DISTRIBUTION \\ IN THE EGG STORED IN O OR 2 PER CENT CARBON DIOXIDE ATMOSPHERE}

Is is known that egg storage is accompanied by a fall of hatchability; in some strains of fowl, this decrease can be reduced by using low rates of carbon dioxide in the atmosphere of the storage room (SAUveur et al., I967). However, nothing is known about the way in which $\mathrm{CO}_{2}$ protects the embryonic cells.

Fertile eggs, laid by three White Leghorn hens, were stored at $12^{\circ} \mathrm{C}$ and $80 \mathrm{p}$. 10o $\mathrm{RH}$ in an atmosphere containing o or $2 \mathrm{p}$. I 100 of $\mathrm{CO}_{2}$. Six eggs for each environment were analyzed after 2 , $\mathrm{I} 2$ and 20 days of storage. The following measurements were made : $\mathrm{pH}, p \mathrm{CO}_{2}$ and osmotic pressure of each part of the egg-white (thick white, inner and outer thin white) ; contents of water, Na, $\mathrm{K}, \mathrm{Ca}, \mathrm{Mg}, \mathrm{Cl}$ and free glucose of the whole white and yolk. Variance analysis were conducted according to factorial methods.

The main results were the following.

I. Figure I-I and table $\mathrm{I}$; mean pH values remained constant (8.2) in the white of the $2 \mathrm{p}$. Ioo. $\mathrm{CO}_{2}$-stored eggs ; moreover the $\mathrm{pH}$ gradient observed in fresh eggs between the three parts of the 
egg white (SAUveuR, I969) remained after 20 days of storage. In op. Ioo. $\mathrm{CO}_{2}$ atmosphere (control group), this $\mathrm{pH}$ gradient disappeared after 2 days of storage ; the mean $\mathrm{pH}$ value increased up to 9.3 after 20 days.

2. Figure I-II and table $\mathrm{I}$ : the partial pressure of $\mathrm{CO}_{2}\left(p \mathrm{CO}_{2}\right)$ in the white was greater in the $\mathrm{CO}_{2}$-stored than in the control eggs (about 20 and $8 \mathrm{~mm} \mathrm{Hg}$ respectively).

3. Figure 2 and table $I$ : the osmotic pressure of the white remained constant in the $\mathrm{CO}_{2}-$ stored eggs. In the control group the osmotic pressure decreased significantly from 260 to $250 \mathrm{mOsm} / \mathrm{r}$ during the first two days of storage, down to a minimum of $242 \mathrm{mOsm} / 1$ at $\mathrm{I} 2$ days.

4. Figure 3 and table $I:$ a transfer of water from the white to the yolk took place during the pre-incubation period ; this transfer seemed to be reduced in the $\mathrm{CO}_{2}$-stored eggs compared to the control.

The content of free glucose in both albumen and yolk was reduced by $I_{5}$ and 56 p. 100 respectively, regardless of the presence of $\mathrm{CO}_{2}$ in the storage atmosphere ; this decrease appeared earlier in the yolk ( 22 days) than in the white (2o days).

5. Figure 4 and table 2 : the most interesting result, in our opinion, was that the $\mathrm{Ca}$ and $\mathrm{Mg}$ contents of the white decreased sharply in the control eggs $(63$ and 2 I p. roo respectively for Ca and $\mathrm{Mg}$ after 20 days). In contrast, these levels did not change in the $\mathrm{CO}_{2}$-stored eggs. This decrease in $\mathrm{Ca}$ and $\mathrm{Mg}$ contents occured in the control eggs at every stage of storage and, probably, did not stop at 20 days. In the same control group of eggs, the $\mathrm{Ca}$ and $\mathrm{Mg}$ contents of the yolk increased but the difference did not reach a significant level of probability because of greater variability.

6. Figures 5 and 6 : the rates of $\mathrm{K}, \mathrm{Na}$, and $\mathrm{Cl}$ either were constant or varied according to the same pattern in the two groups of eggs.

These results are discussed in relation to the physicochemical properties of the egg contents. It is known that the structure of the external layer of the vitellin membrane is controlled by the albumen $\mathrm{pH}$ (Fromm and GAMmon, I968). The current results show that this membrane is as important to ionic transfers as to water transport.

The variations in albumen osmotic pressure are tentatively explained as ruled by electrostatic linkages between divalent cations and the electro-negative groups of the proteins which increase in number with the $\mathrm{pH}$.

\section{RÉFÉRENCES BIBLIOGRAPHIQUES}

Austic R. E., Grau C. R., Matteson G. C., r966. Requirements for glucose and amino acids in defined media for chick embryos. J. Nutr., 90, 175-182.

BaIN J. M., HALL J. M., I969. Observations on the development and structure of the vitelline membrane of the hen's egg : an electron microscope study. Aust. J. Biol. Sci., 22, 653-665.

Bellairs R., Harkness M., Harkness R. D., I963. The vitelline membrane of the hen's egg : a chemical and electron microscopical study. J. Ultrast. Res., 8, 339-359.

BRooks J., PAce J., I938. The distribution of carbon dioxide in the hen's egg. Proc. Roy. Soc. B, 126, I96-209.

Calet C., Blum J.-C., r970. Les vitamines de I'œuf au cours de sa conservation. Ann. Nutr. Alim., 24, B $201-\mathrm{B} 226$.

Cotterill O. J., Winter A. R., 1955. Egg white lysozyme. 3. The effect of pH on the lysozymeovomucin interaction. Poult. Sci., 34, 679-686.

Crolzier G., Sauveur B., I967. Étude des modifications du comportement électrophorétique des protéines de l'albumen au cours de la conservation des cufs de poule. Ann. Biol. anim. Bioch. Biophys. 7, 3I7-32I.

Feeney E., Hill M., I960. Protein chemistry and food research. Adv. Food Res., 10, 23-73.

Fromm D., 1967. Some physical and chemical changes in the vitelline membrane of the hen's egg during storage. J. Food Sci., 32, 52-56.

Fromm D., Gammon S. U., I968. Specific gravity and volume of the hen's egg yolk as influenced by albumen $\mathrm{pH}$ and storage age of the egg. Poult. Sci., 47, rigr-r I96.

Gremy F., Pages J. C., I962. Aspects physiques des phénomenes osmotiques en biologie et en clinique. Rev. franc. Etudes clin. et biol., 7, 648-66o.

Hamm R., 1963. The water inhibiting power of foods. Rec. Adv. Food Sci., 3, 218-229.

Hofmeister F., 1888. Arch. Exp. Pathol. Pharmacol, 24, 247. Cité par Pascal P., r952, Chimie gênérale, tome IV, p. 319, Masson et Cie, Paris. 
JENSEN C., I969. Ultrastructural changes in the avian vitelline membrane during embryonic development. J. Embryol. Exp. Morphol., 21, 467-484.

Kato A., Nakamura R., Sato Y., 1970. Studies on changes in stored shell eggs. IV. Changes in the chemical composition of ovomucin during storage. Agric. Biol. Chem., 34, Ioog-1or3.

Lush I. E., Conchie J., I966. Glycosidases in the egg albumen of the hen, the turkey and the japanese quail. Biochim. Biophys. Acta, 130, 8I-86.

Moran T., r937. Gas storage of eggs. J. Soc. Chem. Indus. Transactions, 61, 96 T-IoI T.

Romanoff A. L., I960. The avian embryo, I92-195, The Macmillan Company, New York.

Sato Y., NAkAmURA R., I959. Studies on changes in stored shell eggs. II. Fractionation of the egg white by chemical method and changes of its constituents during storage. J. Agric. Chem. Soc. Japan., 33, $872-875$.

Sato Y., Nakamura R., Yoshikawa Y., Takagi K., r96o. Studies on changes in stored shell eggs. III. On changes in the albumen minerals during storage. Nippon Nogei Kagaku Kaishi, 34, 1000-1005.

Sauveur B., 1967. Conservation des œufs de poule et éclosivité. Essai de comparaison avec les données obtenues sur la conservation des ceufs de consommation. Ann. Zootech., 16, 89-1r5.

Sauveur B., 1969. Étude de la composition électrolytique des différentes zones de l'albumen de l'œuf chez deux races de poule. Ann. Biol. anim. Bioch. Biophys., 9, 563-573.

Sauveur B., Ferre R., Lacassagne L., I967. Conservation d'œufs de poule sous atmosphère enrichie en gaz carbonique. Action sur les résultats d'éclosion. Ann. Zootech., 16, 35I-356.

Shenstone F. S., I968. The gross composition, chemistry and physico-chemical basis of organization of the yolk and white. In Carter T. C. "Egg quality. A study of the hen's egg. " (B. E. M. B. Symposium $\left.n^{\circ} 4\right), 26-28$, Oliver et Boyd, Edinburgh.

Smith A. J. M., I93I. Rep. Fd. Invest. Bd., Lond., 155-Cité par Brooks J., TAylor D. J., I955. Eggs and egg products. Fd. Invest., Special report, 60.

Spencer J. V., Petribone C. A., Becker W. A., I968. Hatchability of eggs stored in carbon dioxide. Poult. Sci., 46, I721. (Abstr.)

Terepka A. R. Chen P. S. Toribara T. Y., I97o. Ultrafiltration : a conceptual model and a study of sodium, potassium, chloride and water distribution in normal human sera. Physiol. Chem. Phys., 2, $59-78$.

Williams R. J. P., I967. The selectivity of metal-protein interactions. In Peeters H. Protides of the biological fluids, 14, 25-36, EIsevier Publish. Co., Amsterdam.

YoN J., I969. Structure et dynamique conformationnelle des protéines, p. 157, Hermann, Paris. 